\title{
The efficacy of iodine-125 permanent brachytherapy versus intensity-modulated radiation for inoperable salivary gland malignancies: study protocol of a randomised controlled trial
}

\author{
Shu-Ming Liu' ${ }^{1}$, Hai-Bo Wang ${ }^{2}$, Yan Sun ${ }^{3}$, Yan Shi ${ }^{1}$, Jie Zhang ${ }^{1}$, Ming-Wei Huang ${ }^{1}$, Lei Zheng ${ }^{1}$, Xiao-Ming Lv'
} Bao-Min Zheng ${ }^{3}$, Kathleen H. Reilly ${ }^{4}$, Xiao-Yan Yan ${ }^{2}$, Ping Ji ${ }^{2}$, Yang-feng $\mathrm{Wu}^{2}$ and Jian-Guo Zhang ${ }^{*}$

\begin{abstract}
Background: Radiation therapy is the method of choice for subjects with inoperable salivary gland malignancies. I125 brachytherapy, delivering a high radiation dose to a tumor but sparing surrounding normal tissues, is supposed to be ideal modality for the treatment of salivary gland malignancies. We designed a randomised controlled clinical trial to compare the efficacy of I-125 permanent brachytherapy (PBT) versus intensity-modulated radiation therapy (IMRT) for inoperable salivary gland malignancies.

Methods/Design: In this study, inclusion criteria are subjects with inoperable salivary gland malignancies, aged 1880 years, have provided informed consent, with at least one measurable tumor focus, be able to survive $\geq 3$ months, Karnofsky performance status $\geq 60$, have adequate hematopoietic function of bone marrow, have normal liver and kidney function, and are willing to prevent pregnancy.

Exclusion criteria include a history of radiation or chemotherapy, a history of other malignant tumors in the past 5 years, receiving other effective treatments, participating in other clinical trials, with circulatory metastasis, cognitive impairment, severe cardiovascular and cerebrovascular diseases, acute infection, uncontrolled systemic disease, history of interstitial lungdisease, and being pregnant or breast feeding.

The study will be conducted as a clinical, prospective, randomised controlled trial with balanced randomisation (1:1). The planned sample size is 90 subjects. Subjects with inoperable salivary gland malignancies are randomised to receive either I-125 PBT or IMRT, with stratification by tumor size and neck lymph node metastasis. Participants in both groups will be followed up at 2, 4, 6, 9, 12, 15, 18, 21 and 24 months after randomization. The primary outcome is local control rate of the primary site (based on imaging findings and clinical examination, RECIST criteria) in 1 year. Secondary outcomes are progression-free survival, overall survival, quality of life (QOL) measured with the European Organization for Research and Treatment of Cancer QOL Questionnaire (EORTC QLQ-C30 and QLQ-H\&N35) of Chinese version, and safety of treatment. Chi-squared test is used to compare the local control rates in both groups. The survival curves are estimated by the Kaplan-Meier method, and log-rank test is used to test the significant difference.

(Continued on next page)
\end{abstract}

\footnotetext{
*Correspondence: rszhang@126.com

Shu-Ming Liu and Hai-Bo Wang are co-first authors.

'Department of Oral and Maxillofacial Surgery, Peking University School and

Hospital of Stomatology, 22 Zhongguancun South St, Haidian Dist, Beijing.

100081, PR China

Full list of author information is available at the end of the article
}

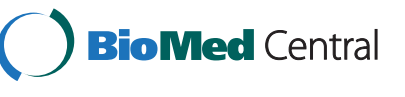

(c) 2016 Liu et al. Open Access This article is distributed under the terms of the Creative Commons Attribution 4.0 International License (http://creativecommons.org/licenses/by/4.0/), which permits unrestricted use, distribution, and reproduction in any medium, provided you give appropriate credit to the original author(s) and the source, provide a link to the Creative Commons license, and indicate if changes were made. The Creative Commons Public Domain Dedication waiver (http://creativecommons.org/publicdomain/zero/1.0/) applies to the data made available in this article, unless otherwise stated. 
(Continued from previous page)

Discussion: Only few observational studies have investigated the effect of I-125 PBT on inoperable salivary gland malignancies. To our knowledge, this is the first randomised controlled trial to investigate the efficacy of I-125 PBT for subjects with inoperable salivary gland malignancies, and will add to the knowledge base for the treatment of these subjects.

Trial registration: The study is registered to Clinical Trials.gov (NCT02048254) on Jan 29, 2014.

Keywords: I-125 permanent brachytherapy, Intensity-modulated radiation therapy, Inoperable salivary gland malignancy, Local control rate, Quality of life

\section{Background}

Primary malignant tumors of the major and minor salivary glands are relatively rare entities, accounting for only about $3-5 \%$ of all head and neck malignancies. They also contain a diverse group of histologies, with disparate characteristics in terms of aggressiveness and patterns of spread [1]. Surgery has historically been the mainstay of treatment for salivary gland malignancies. Subjects with low-grade tumors are usually treated with surgery alone if complete excision can be achieved. Radiotherapy, as a postoperative adjunct to surgery, has traditionally been reserved for subjects with microscopically high-grade tumors, positive margins or nerve invasion. Although carcinomas originating from the salivary glands were previously thought to be radioresistant, the role of adjunct radiotherapy in therapy has become well recognized [2, 3]. However, the therapy for inoperable malignant salivary has been extremely challenging in clinical practice: some subjects are either not candidates for definitive resection or undergo limited procedures leaving behind gross residual disease. Typically, these subjects are deemed inoperable because of technical issues related to the extensiveness or location of the primary tumor. Another subset of inoperable subjects present with medical comorbidities that places them at unacceptably high risk for perioperative complications. Lastly, some subjects refuse surgical therapy out of personal preference. For whatever reason, these inoperable subjects have all traditionally been offered definitive radiation therapy as an alternative to surgery. Unfortunately, the reported results following low linear energy transfer (LET) irradiation are poor, with overall local control rates average below $30 \%$ [4-10]. In a multicenter randomised controlled study conducted by the American Radiation Therapy Oncology (RTOG) and the UK Medical Research Council (MRC), the 2-year local control rate (LCR) of unresectable salivary gland cancers with conventional external radiotherapy was only $17 \%$ [10].

The dose response of biological systems is influenced by the LET of ionizing radiation. In general, relative radiobiological effectiveness (RBE) increases with LET. A number of studies have been carried out to investigate the effectiveness of high-LET RT such as fast neutron RT. Batterman et al. described an elevated RBE for fast neutrons in the treatment of lung metastases of malignant salivary gland tumors. In this study, the highest RBE values up to 8 were found for ACC [11]. The randomised controlled study conducted by RTOG and MRC showed that the 2-year local control rate was $67 \%$ for fast neutron radiotherapy compared with $17 \%$ for conventional photon radiation [10]. However, the indication of fast neutron radiotherapy has been strictly limited because of its unacceptable damage to the surrounding normal tissues $[12,13]$. In heavy particles radiotherapy, carbon ions have similar radiobiologic properties as neutrons, and higher RBE values can be expected for salivary gland tumors. Compared with neutron RT, carbon ions additionally provide physical selectivity due to an inverse dose profile. Thus, carbon ions was considered to have potentially greater clinical value [14]. In Germany, intensitymodulated radiation therapy (IMRT) combined with carbon ions radiotherapy was recommended as the standard treatment for inoperable salivary gland malignancies [15]. But carbon ions radiotherapy was expensive and unavailable, which obviously limits its application.

Brachytherapy is an important modality in the treatment of human malignancy with ionizing radiation. Where applicable, it may be the method of choice for the following reasons $[16,17]$. First, the localized dose distribution enhances the ratio of tumor dose to surrounding normal tissue dose. Second, the reduction of oxygen enhancement ratio and dose rate may partially circumvent the radioresistance of hypoxic tumor cells. Permanent implants of I125 sources in focus sites have been widely used, especially for prostatic cancer [18]. Because LET increases with decreasing photon energy, I-125 source with low energy photons(average $28 \mathrm{keV}$ ) has higher RBE values (approximately 1.4) [19]. In addition, the low photon energy also provides more sparing for adjacent normal tissue and easy resolution of the problem of protecting medical staff from radiation exposure. Some studies have shown that I-125 permanent brachytherapy (PBT) may have potential advantages in local control of salivary gland malignancies and in minimizing radiobiological damage to normal adjacent tissues [20-24]. I-125 has a long half-life of 60 days and may be ineffective in eradicating tumors with fast growth kinetics [25-27]. The clinical efficacy of I-125 in 
prostatic cancer may be due to the relative slow proliferative rate of this disease [25-27]. Like prostatic cancer, many of salivary gland malignancies are characteristically slowly proliferating tumours with long natural histories. Therefore, I-125 is supposed to be ideal modality for the treatment of salivary gland malignancies, as suggested by other nonrandomised clinical trials. In order to obtain more credible evidence, we launch a Phase III, randomised controlled trial to compare the efficacy of I-125 PBT versus IMRT for inoperable salivary gland malignancies.

\section{Methods and design}

\section{Trial design and setting}

The study is conducted as a single center, prospective, randomised controlled trial with balanced randomisation (1:1) for subjects who have inoperable malignant salivary gland tumors are randomised to receive either I-125 PBT or IMRT. Subjects are stratified by tumor size $(\leq 4 \mathrm{~cm}$ vs. $>4 \mathrm{~cm}$ ) and neck lymph node metastasis (yes vs. no). Subjects are recruited continuously by oncologists at Department of Oral and Maxillofacial Surgery, Peking University School and Hospital of Stomatology which is the only department to treating salivary gland malignancies by I-125 PBT in China.

\section{Ethical approval}

The protocol and informed consent form have been reviewed and approved by the Institutional Review Boards of the Peking University Health Science Center in Beijing, China, and registered at www.clinicaltrials.gov (NCT02048254). Figure 1 illustrates the flow diagram of the study for both the intervention and control groups.

\section{Participants}

Ninety subjects will be recruited for the study and divided into an intervention group (I-125 PBT) and a control group (IMRT).

\section{Inclusion criteria}

In the study, inclusion criteria are as follows: (1) willing to participate and sign informed consent; (2) aged 18-80 years; (3) malignancies originating from major or minor salivary glands based on pathological and/or cytological diagnosis, including primary or recurrent tumors; (4) with inoperable tumors, including unresectable primary locally advanced tumors; unresectable recurrent tumors; and unable to undergo surgery due to other medical comorbidities or refusal of surgery out of personal preference, and being T3/T4 T-stage; (5) with at least one

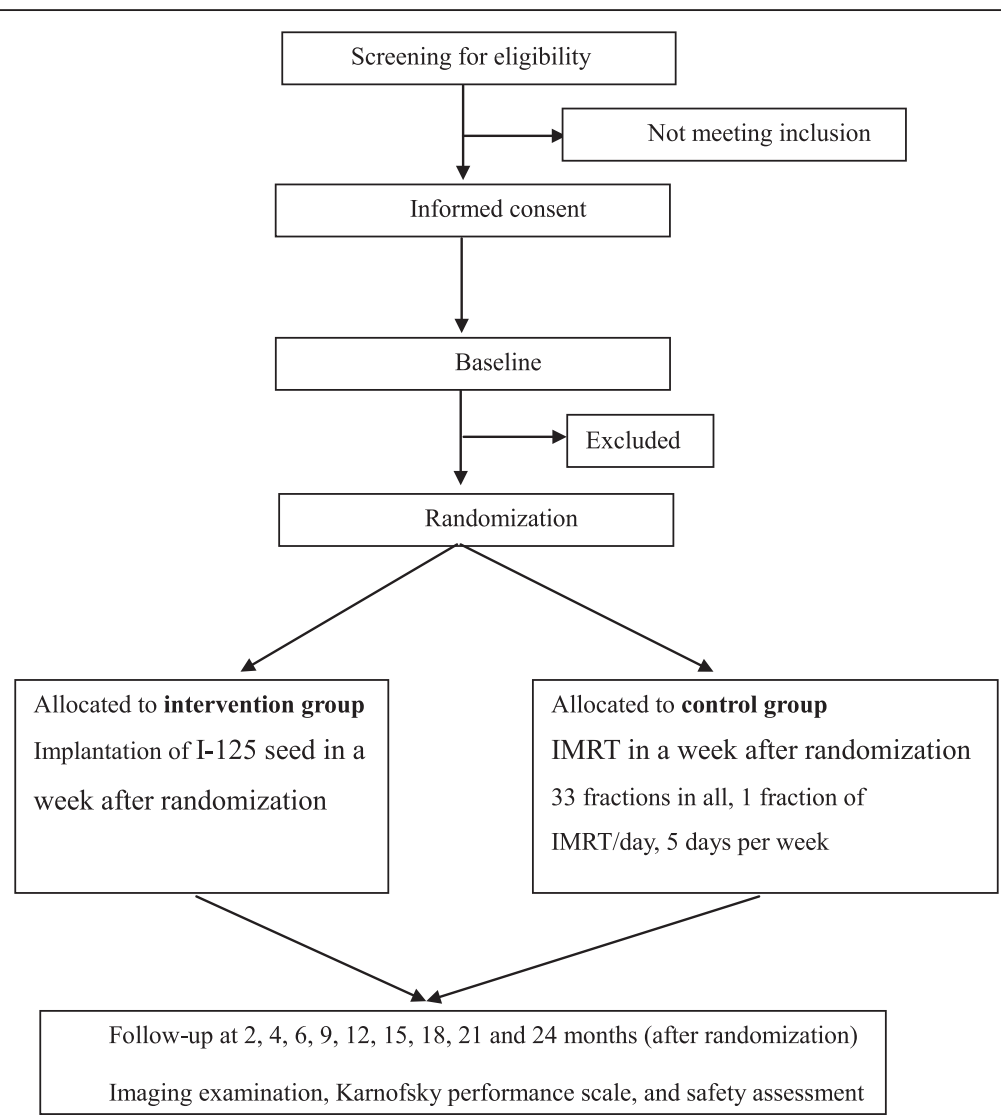

Fig. 1 Study flow chart 
measurable tumor focus based on RECIST criteria and imaging diagnosis completed in 30 days before enrollment; (6) expected survival time $\geq 3$ months; (7) Karnofsky performance status $\geq 60$; (8) adequate hematopoietic function of bone marrow in previous 7 days: hemoglobin $\geq 9 \mathrm{~g} / \mathrm{dL}$, white blood cell count $\geq 3.0 \times 10^{9} / \mathrm{L}$, neutrophils count $\geq 1.5 \times 10^{9} / \mathrm{L}$, platelet count $\geq 100 \times 10^{9} / \mathrm{L}$; (9) normal liver and kidney function in previous 14 days: total bilirubin in serum $\leq 1.5$ times the upper limit of normal (ULN), alanine transaminase and aspartate transaminase $\leq 3$ times ULN, creatinine $\leq 1.5$ times ULN; (10) willing to take measures to prevent pregnancy.

\section{Exclusion criteria}

Exclusion criteria include: (1) with a history of radiation treatment on head and neck; (2) with a history of other malignant tumors in past 5 years, except for healed skin basal cell carcinoma and cervical carcinoma in situ; (3) with a history of tumor chemotherapy; (4) receiving other effective treatments; (5) having participated in other clinical trials in 4 weeks before enrollment; (6) with circulatory metastasis; (7) with the histology subtype of squamous cell carcinoma; (8) without measurable tumor focus, such as diffuse infiltrative carcinoma; (9) cognitive impairment due to neursis or psychosis; (10) cardiovascular and cerebrovascular diseases with clinical significance, such as heart failure in NYHA III/IV, uncontrolled coronary heart disease, myocardiopathy, uncontrolled arrhythmia, uncontrolled hypertension, history of myocardial infarction or cerebral infarction in the past half year; (11) severe clinical infection in 14 days before randomization including active pulmonary tuberculosis; (12) human immunodeficiency virus infection, active hepatitis B or hepatitis C; (13) uncontrolled systemic disease, such as diabetes mellitus; (14) with the history of interstitial lung disease, such as interstitial pneumonia, pulmonary fibrosis, or diagnosed as interstitial lung disease by chest Xray/CT image; (15) being pregnant or breast feeding.

\section{Withdrawal of individual subjects}

Subjects can withdraw from the study at any time for any reason without any consequences. The investigator can decide to let a subject out from the study for particular medical reasons, for example, serious adverse events. For every subject who decides to withdraw from the study, the reasons for withdrawal should be recorded.

\section{Randomization}

Central randomization based on interactive web response system (IWRS,Brightech Clinical Information Management System) is carried out by Peking University Clinical Research Institute, which is independent of the trial administration office. The allocation sequence is computergenerated 1:1 with dynamic randomization system and is stratified by tumor size and neck lymph node metastasis.

\section{Blinding}

Allocation status cannot be blinded for the participants and investigators due to different treatment methods and the visibility of implanted I-125 seed in CT image. However, the primary outcome (response to treatment) will be evaluated by an independent assessment board. Further, all statistical analysis will be done by a statistician in Peking University Clinical Research Institute who is not affiliated with the trial.

\section{Intervention -implanationt of I-125 seed}

All subjects who are assigned to the intervention group will receive I-125 seed permanent implantation in the study. The operation of I-125 seed implantation will be conducted at the department of Oral and Maxillofacial Surgery, Peking University School and Hospital of Stomatology. I-125 seeds will be ordered from the manufacturer 1-2 days before surgical operation. The placement of the I-125 seed was determined from CT scans with the use of a brachytherapy treatment planning system (BTPS; Beijing Atom and High Technique Industries, Beijing, China). The I-125 seed (model 6711; Jaco Pharmaceuticals Co. Ltd., Zhejiang, China) activity was $0.9-1.0 \mathrm{U}$ per seed and had a half-life of 59.6 days. Clinical target volume (CTV) is defined as gross tumor volume (GTV) and its surrounding potential sub-clinical focus or microscopic focus, and it is also divided as CTV1 and CTV2. CTV2, namely high risk area, is defined as primary tumor and around $10 \mathrm{~mm}$, as well as drainage regions of involved lymph node. CTV2, namely low risk area, is defined at 5 to $10 \mathrm{~mm}$ beyond CTV2 and lymphatic drainage area which should be prevented by irradiation. The prescribed dose is 120 Gy for CTV1 and 140 Gy for CTV2. And it is commonly assumed that irradiation dose can accumulate to prescribed dose in 6 months.

A CT scan is obtained one week after seeds implantation. The CT images in combination with BTPS are used to detect the location, number and isodose plot distribution of seeds. Re-implantation can be considered if implant related deficiencies are identified, including asymmetrical distribution, shedding or movement.

\section{Control group - IMRT}

All subjects who are assigned to the control group will receive IMRT in the study, which is done at the department of radiotherapy, Peking University Cancer Hospital \& Institute. Prescribed dose for planning target volume and dose segmentation is computed with simultaneous integrated boost modulated radiation therapy. In each fraction of irradiation treatment, a specific dose is used for different target volume during the entire course of treatment. Totally, 70 Gy IMRT in 33 fractions (5 fractions per week) are prescribed to the GTV, $60 \mathrm{~Gy} / 33$ fractions to CTV2, and 56 Gy/33 fractions to CTV1. 
Initial screening, assessment and follow-up

After providing informed consent, potential participants will be asked standardized questions about their demographic characteristics and medical history. In addition, physical examination, electrocardiography, routine urine test, blood clotting function, urine $\beta$-human chorionic gonadotropin (HCG) for reproductive-age women, specialized examination, imaging examination, blood routine examination and biochemistry, pathological diagnosis and Karnofsky performance scale etc. will also be done and used for checking inclusion/exclusion criteria (Fig. 2).
After randomization, eligible participants will receive their treatment planning investigations and are followedup following the same schedule for both intervention group and control group (Fig. 2) until recurrence, other malignancy or death is confirmed. Treatment duration is approximately 6-7 weeks for IMRT group. All the participants in both groups will be followed up at 2, 4, 6, 9, 12, 15, 18, 21 and 24 months after randomization, with specialized examination, imaging examination, blood routine examination and biochemistry, Karnofsky performance scale, recording of treatment and adverse events assessment done

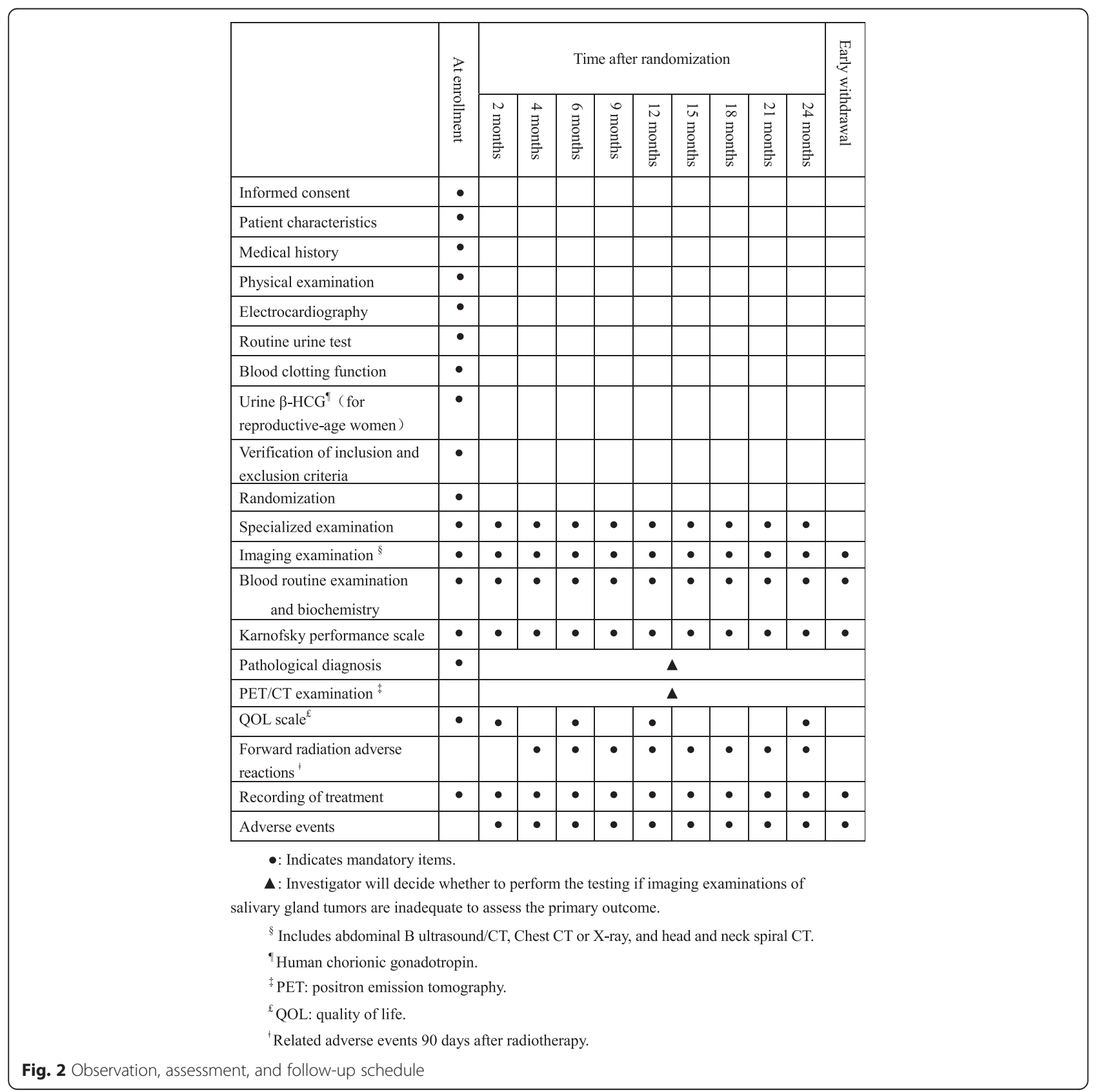


each time. Quality of life (QOL) scale will be assessed at 2, 6,12 , and 24 months. Forward radiation adverse reactions will be evaluated at each follow-up appointment except at 2 months after randomization. In addition, pathological diagnosis and PET/CT examination will be considered by the investigator if imaging examinations are inadequate to assess the primary outcome. Radioactive adverse events were classified by The National Cancer Institute-Common Terminology Criteria for Adverse Events (NCI-CTCAE) 4.0 standard. Chronic radioactive damages were classified by the criteria of Radiation Therapy Oncology Group/ European Organization for Research and Treatment of Cancer (RTOG/EORTC), with common signs and severity recorded.

\section{Outcomes}

Assessment of efficacy will be carried out by evaluation of imaging examination at each follow-up. If applicable, response to treatment will be evaluated according to the RECIST criteria (version 1.1) and classified as complete response $(\mathrm{CR})$, partial response $(\mathrm{PR})$, stable disease $(\mathrm{SD})$ and progressive disease (PD).

The primary outcome is estimated as the difference between the intervention and the control group in local control rate in 1 year. Local control rate is judged to have been attained if there is no evidence of $\mathrm{PD}$ at the primary site based on imaging findings and clinical examination at follow-up. However, distant metastasis and second primary cancer are not belonged to $\mathrm{PD}$ at the primary site in accordance with RECIST criteria (version 1.1) [28].

Secondary outcomes are a) progression-free survival (PFS), defined as the time from randomization to the earliest occurrence of PD in whole body or death due to any cause; PD in whole body includes $\mathrm{PD}$ at the primary site, distant metastasis and second primary cancer; b) overall survival (OS), from the date of randomization to the date of death from any cause or last date when the participant is alive; c) QOL evaluated using the European Organization for Research and Treatment of Cancer QOL Questionnaire (EORTC QLQC30 and QLQ-H\&N35) of Chinese version; and d) safety of treatment.

\section{Sample size estimation}

Based on medical record of subjects with inoperable salivary gland malignancies in Peking University Cancer Hospital \& Institute and in Peking University School and Hospital of Stomatology, local control rate in 1 year was $81 \%$ among subjects with I-125 PBT, and $45 \%$ among subjects with IMRT. Based on a difference of $36 \%$ between groups on the primary outcome, a total of 72 participants are required to provide $90 \%$ power, with the use of two-sided significance level of 0.05. Assuming a $20 \%$ drop-out rate, approximately 90 subjects (or 45 subjects per arm) will be enrolled.

\section{Statistical analysis}

Analyses will be made using SAS statistical software (version 9.3, SAS Institute, Cary, NC, USA) by researchers at the Peking University Clinical Research Institute. The primary analyses will be done on an intentto-treat basis and the last observation carry forward (LOCF) is used for missing values. Descriptive statistics will be used to summarize demographic and clinical characteristics of subjects randomised to the intervention and control group. The difference between two groups on demographic and clinical characteristics, response rate and drop-out rate will be compared using ttests (or Wilcoxon rank sum test) and chi-square test/ Fisher's exact test as appropriate.

Primary outcome (local control rate in 1 year) analyses will be carried out using chi-squared tests. The survival curves (PFS and OS) are estimated by the Kaplan-Meier method, and log-rank test is used to test the null hypothesis that the respective curves are equal between the two groups. A two-sided significance level of $5 \%$ is used. A covariance model will be used to compare the QOL between two groups by adjusting the difference of baseline. The incidence of adverse events between two groups is compared with the chi-square test/Fisher's exact test.

\section{Discussion}

This trail is conducted to prospectively evaluate I-125 PBT for inoperable salivary gland malignancies in terms of the efficacy, safety and the QOL.

Few studies have investigated the effect of I-125 PBT for inoperable salivary gland malignancies and thus, the evidence regarding I-125 PBT for inoperable salivary gland malignancies is sparse. In recent years, we have been exploring the effectiveness and feasibility of I-125 PBT for inoperable salivary gland malignancies. Our previous studies displayed that I-125 PBT may be one of the most promising treatment for inoperable salivary gland malignancies, but the evidence of these results is not sufficient enough due to limited number of observational studies [23, 24]. The proposed study is a randomised controlled single-centre trial conducted among subjects with inoperable salivary gland malignancies. To our knowledge, it is the first trial to investigate the efficacy of I-125 PBT for subjects with inoperable salivary gland malignancies, and will add to the knowledge base in a number of ways. Not only tumor cells, but also normal cells, are simultaneously killed by radiotherapy. Many side effects are often observed during and after radiotherapy, including lower white blood cell count, general weakness and loss of appetite, mucositis, xerostomia, hearing loss, radiation dermatitis, fibrosis, osteoradionecrosis of the mandible, and 
injury to optic apparatus. These side effects may lower the QOL of these subjects. In addition to effectiveness, we also investigate safety and QOL. These findings will help to systematically evaluate clinical application value of I-125 PBT.

We set a comparison treatment as the control arm, which will help us to assess the efficacy of I-125 PBT. We choose IMRT as the control arm because of its fewer side effects. IMRT is more precise radiotherapy modality which helps to reduce normal tissue damages compared with conventional external radiotherapy. Although there was no evidence that IMRT could significantly improve the LCR, many studies had shown that IMRT could significantly reduce toxic side effects when used in the head and neck cancer region [29]. This IMRT comparison arm can help to protect patient and improve compliance.

On account of the low prevalence, we will recruit patient with relatively inclusive entry criteria, and anticipate recruiting subjects across the spectrum of histologies and from various inoperable circumstances. These features will improve the generalisability of our findings. On the other hand, subjects will constitute a heterogeneous group, and this could weaken the power of our trial. We have taken a number of steps to reduce selection bias. The trial does not include all relevant subjects (e.g., some subjects are excluded, such as with subtotal resection, T1/T2-staged, and metastatic cancer). In addition, subjects are stratified by tumor size $(\leq 4 \mathrm{~cm}$ vs. $>4 \mathrm{~cm})$ and neck lymph node metastasis (yes vs. no) to balance random allocation.

The treatment allocation cannot be blinded for participants and study staffs due to different procedures and the visibility of implanted I-125 seed in CT image, which is of course a limitation. However, to reduce observer bias in assessment, all assessment data are collected by research assistants who did not participate in the study; in addition, the primary outcome (response to treatment) will be evaluated by independent assessment board. Further, all statistical analysis will be done by a statistician at Peking University Clinical Research Institute who is not affiliated with the trial.

\section{Conclusion}

The proposed study aims to investigate whether subjects with inoperable salivary gland malignancies will benefit from iodine-125 seed permanent brachytherapy. We will also study the side effects of such treatment.

\section{Competing interests}

The authors declare that they have no competing interests.

\section{Authors' contributions}

SML, HBW, SY, XYY, PJ, YFW and JGZ contribute to the development of the study protocol. JGZ is the principal investigator and managed the protocol. $S M L, H B W, K H R, X Y Y, P J$ and $Y F W$ are involved in the initial draft of the manuscript and writing it. SY, YS, JZ, MWH, LZ, XML and BMZ are responsible for participant enrollment, follow-up and data entry. HBW, SY, YS, JZ, MWH, $L Z, X M L, B M Z, K H R, X Y Y, P J, Y F W$ and JGZ are involved in reviewing the manuscript. All authors read and approve the final manuscript.

\section{Acknowledgements}

We wish to thank the following project nurses for collecting the data: Fu Chen, Peking University Cancer Hospital \& Institute; Li Wei, Xiao-Jing Li, Peking University School and Hospital of Stomatology. Furthermore, we wish to thank the IMRT teams for participating in the trial. Last, but not least, we wish to thank the subjects for their participation.

\section{Funding}

This research is funded by Peking University Clinical Research Program (grant number, PUCRP201308) in Peking University Health Science Center. Excess treatment and service support costs incurred by the research are funded by the Peking University School and Hospital of Stomatology.

\section{Author details}

${ }^{1}$ Department of Oral and Maxillofacial Surgery, Peking University School and Hospital of Stomatology, 22 Zhongguancun South St, Haidian Dist, Beijing 100081, PR China. ${ }^{2}$ Peking University Clinical Research Institute, Xueyuan Rd 38\#, Haidian Dist, Beijing 100191, PR China. ${ }^{3}$ Department of radiotherapy, Peking University Cancer Hospital \& Institute, 52 Fucheng Road, Haidian Dist, Beijing 100142, PR China. ${ }^{4}$ Independent Consultant, New York, NY, USA.

Received: 12 August 2014 Accepted: 3 March 2016

Published online: 07 March 2016

\section{References}

1. Day TA, Deveikis J, Gillespie MB, Joe JK, Ogretmen B, Osguthorpe JD, Reed SG, Richardson MS, Rossi M, Saini R et al. Salivary gland neoplasms. Curr Treat Options Oncol. 2004;5(1):11-26.

2. Mendenhall WM, Morris CG, Amdur RJ, Werning JW, Villaret DB. Radiotherapy alone or combined with surgery for salivary gland carcinoma. Cancer. 2005;103(12):2544-50.

3. Terhaard $\mathrm{CH}$, Lubsen $\mathrm{H}$, Rasch CR, Levendag PC, Kaanders HH, Tjho-Heslinga RE, van Den Ende PL, Burlage F, Dutch H, Neck Oncology Cooperative G. The role of radiotherapy in the treatment of malignant salivary gland tumors. Int J Radiat Oncol Biol Phys. 2005;61(1):103-11.

4. Borthne A, Kjellevold K, Kaalhus O, Vermund H. Salivary gland malignant neoplasms: treatment and prognosis. Int J Radiat Oncol Biol Phys. 1986; 12(5):747-54.

5. Elkon D, Colman M, Hendrickson FR. Radiation therapy in the treatment of malignant salivary gland tumors. Cancer. 1978;41(2):502-6.

6. Fu KK, Leibel SA, Levine ML, Friedlander LM, Boles R, Phillips TL. Carcinoma of the major and minor salivary glands: analysis of treatment results and sites and causes of failures. Cancer. 1977:40(6):2882-90.

7. Rafla S. Malignant parotid tumors: natural history and treatment. Cancer. 1977:40(1):136-44

8. Reddy EK, Mansfield CM, Hartman GV, Rouby E. Malignant salivary gland tumors: role of radiation therapy. J Natl Med Assoc. 1979;71(10):959-61.

9. Shidnia H, Hornback NB, Hamaker R, Lingeman R. Carcinoma of major salivary glands. Cancer. 1980;45(4):693-7.

10. Griffin TW, Pajak TF, Laramore GE, Duncan W, Richter MP, Hendrickson FR, Maor MH. Neutron vs photon irradiation of inoperable salivary gland tumors: results of an RTOG-MRC Cooperative Randomized Study. Int J Radiat Oncol Biol Phys. 1988;15(5):1085-90.

11. Battermann JJ, Breur K, Hart GA, van Peperzeel HA. Observations on pulmonary metastases in patients after single doses and multiple fractions of fast neutrons and cobalt-60 gamma rays. Eur J Cancer. 1981;17(5):539-48.

12. Laramore GE, Krall JM, Griffin TW, Duncan W, Richter MP, Saroja KR, Maor MH, Davis LW. Neutron versus photon irradiation for unresectable salivary gland tumors: final report of an RTOG-MRC randomized clinical trial. Radiation Therapy Oncology Group. Medical Research Council. Int J Radiat Oncol Biol Phys. 1993;27(2):235-40.

13. Huber PE, Debus J, Latz D, Zierhut D, Bischof M, Wannenmacher M, Engenhart-Cabillic R. Radiotherapy for advanced adenoid cystic carcinoma: neutrons, photons or mixed beam? Radiother Oncol. 2001;59(2):161-7.

14. Schulz-Ertner D, Nikoghosyan A, Jakel O, Haberer T, Kraft G, Scholz M, Wannenmacher M, Debus J. Feasibility and toxicity of combined photon and carbon ion radiotherapy for locally advanced adenoid cystic carcinomas. Int J Radiat Oncol Biol Phys. 2003;56(2):391-8.

15. Jensen AD, Nikoghosyan A, Windemuth-Kieselbach C, Debus J, Munter MW. Combined treatment of malignant salivary gland tumours with intensity- 
modulated radiation therapy (IMRT) and carbon ions: COSMIC. BMC Cancer. 2010;10:546.

16. Hall EJ. Radiation dose-rate: a factor of importance in radiobiology and radiotherapy. Br J Radiol. 1972;45(530):81-97.

17. Ling CC, Spiro IJ, Mitchell J, Stickler R. The variation of OER with dose rate. Int J Radiat Oncol Biol Phys. 1985;11(7):1367-73.

18. Langley SE, Laing RW. lodine seed prostate brachytherapy: an alternative first-line choice for early prostate cancer. Prostate Cancer Prostatic Dis. 2004;7(3):201-7.

19. Ling CC, Li WX, Anderson LL. The relative biological effectiveness of I-125 and Pd-103. Int J Radiat Oncol Biol Phys. 1995;32(2):373-8.

20. Glaser MG, Leslie MD, Coles I, Cheesman AD. lodine seeds in the treatment of slowly proliferating tumours in the head and neck region. Clin Oncol. 1995;7(2):106-9.

21. Zhanf JG, Zhang J, Song TL, Zheng L, Cai ZG, Yu GY. Preliminary study of treating parotid gland malignant tumor with saving facial nerve (surgery associated with 125 I seed implant brachytherapy). Zhonghua Kou Qiang Yi Xue Za Zhi. 2008:43(3):132-5.

22. Zhang J, Zhang JG, Song TL, Zhen L, Zhang Y, Zhang KH, Yang ZH, Yu GY. 1251 seed implant brachytherapy-assisted surgery with preservation of the facial nerve for treatment of malignant parotid gland tumors. Int J Oral Maxillofac Surg. 2008;37(6):515-20.

23. Zhang J, Zhang JG, Cai ZG, Zhang Y, Huang MX, Yu GY. 125 I seed implant brachytherapy of recurrent parotid gland malignant tumor. Zhonghua Kou Qiang Yi Xue Za Zhi. 2009:44(1):2-4

24. Zheng L, Zhang J, Song T, Zhang J, Yu G, Zhang Y. 125 l seed implant brachytherapy for the treatment of parotid gland cancers in children and adolescents. Strahlenther Onkol. 2013;189(5):401-6.

25. Arslan NC, Geard CR, Hall EJ. Low dose-rate effects of cesium-137 and iodine-125 on cell survival, cell progression, and chromosomal alterations. Am J Clin Oncol. 1986;9(6):521-6.

26. Marchese MJ, Hall EJ, Hilaris BS. Encapsulated iodine-125 in radiation oncology. I. Study of the relative biological effectiveness (RBE) using low dose rate irradiation of mammalian cell cultures. Am J Clin Oncol. 1984;7(6):607-11.

27. Ling CC. Permanent implants using Au-198, Pd-103 and I-125: radiobiological considerations based on the linear quadratic model. Int J Radiat Oncol Biol Phys. 1992;23(1):81-7.

28. Eisenhauer EA, Therasse P, Bogaerts J, Schwartz LH, Sargent D, Ford R, Dancey J, Arbuck S, Gwyther S, Mooney M et al. New response evaluation criteria in solid tumours: revised RECIST guideline (version 1.1). Eur J Cancer. 2009;45(2):228-47.

29. Bhide SA, Newbold KL, Harrington KJ, Nutting CM. Clinical evaluation of intensity-modulated radiotherapy for head and neck cancers. Br J Radiol. 2012;85(1013):487-94.

\section{Submit your next manuscript to BioMed Central and we will help you at every step:}

- We accept pre-submission inquiries

- Our selector tool helps you to find the most relevant journal

- We provide round the clock customer support

- Convenient online submission

- Thorough peer review

- Inclusion in PubMed and all major indexing services

- Maximum visibility for your research

Submit your manuscript at www.biomedcentral.com/submit

) Biomed Central 